\title{
Vote selection mechanisms and probabilistic data association-based mobile node localization algorithm in mixed LOS/NLOS environments
}

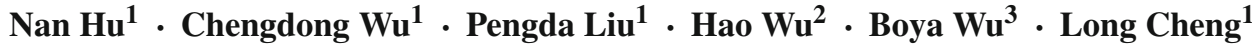

Published online: 22 October 2015

(C) The Author(s) 2015. This article is published with open access at Springerlink.com

\begin{abstract}
As one of the key technologies of wireless sensor networks (WSNs), the localization of mobile nodes (MN) is one of the most significant research topics in WSNs. When a line-of-sight (LOS) channel is available, accuracy localization result can be obtained. Motivated by the fact that the non-line-of-sight (NLOS) propagation of signal is ubiquitous and decreases the accuracy of localization, we propose a MN localization algorithm in mixed LOS/NLOS environments. Considering the characteristics of NLOS error, we propose a localization algorithm based on vote selection mechanisms to filter the distance measurements and reserve the reliable measurements. Then a modified probabilistic data association algorithm is proposed to fuse the multiple measurements reserved from the vote selection. The position of the $\mathrm{MN}$ is finally determined by a linear least squares algorithm based on reference nodes selection. This algorithm effectively mitigates various kinds of NLOS errors and largely improves the localization accuracy of the MN in mixed LOS/NLOS environments. The simulation and experiments results show that the proposed algorithm has better robustness and higher localization accuracy than other methods.
\end{abstract}

Electronic supplementary material The online version of this article (doi:10.1007/s11235-015-0100-z) contains supplementary material, which is available to authorized users.

$\triangle \mathrm{Nan} \mathrm{Hu}$

neuhunan0806@gmail.com

$凶$ Pengda Liu

duozckchlz@126.com

1 College of Information Science and Engineering, Northeastern University, Shenyang 110819, China

2 Engineering Faculty, University of Sydney, Sydney, NSW 2006, Australia

3 School of Foreign Languages, Dalian Maritime University, Dalian 116026, China
Keywords Wireless sensor networks · Non-line-of-sight · Localization - Vote mechanisms - Probabilistic data association

\section{Introduction}

Wireless sensor networks (WSNs) have attracted significant attention in recent years. Sensors have been used widely in different applications such as environment, health, military, national security and other fields. Recently, localization of mobile nodes (MNs) in WSNs has received considerable attention [1-4]. Global Positioning System and Cellular Positioning System are the most common localization systems, but their performance deteriorate for indoor localization [5].

The common methods to estimate the distance between a transmitter and a receiver are received signal strength indicator, time of arrival, time difference of arrival and angle of arrival [6]. Most of the papers assume that the signal propagation is line of sight (LOS) between the $\mathrm{MN}$ and the beacon nodes $(\mathrm{BN})$. We can obtain an accurate distance estimation by using the filtering techniques [7]. However, the direct propagation path between the $\mathrm{MN}$ and the $\mathrm{BN}$ may be blocked by obstacles in practical applications of WSNs, such as the indoor and urban environments. Non-line of sight (NLOS) signal propagation is often inevitable due to the reflection and diffraction. The distance measurement error includes the measurement noise and the NLOS propagation error. Therefore the NLOS error is one of the challenges for accurate mobile location estimation in WSNs.

Methods for mitigating negative impact from NLOS in existing localization algorithms fall into two major categories:

Some researchers perform LOS reconstruction with the distance measurement. They mitigate the NLOS error of 
the distance measurements and make the revised measurements closer to the real distance measurements, then apply the localization algorithm to accomplish the locating. Wylie [8] finds the $N$ th order polynomial fit to smooth the data to correct the error. Based on the premise that standard deviation from the measured value is greater in NLOS than in LOS, this method determines whether the measured value contains NLOS error by comparing standard deviation of the actual measured value. Then the LOS reconstruction is performed on NLOS distance measurements. Communication will lead to increase in energy consumption because the method requires statistical properties of the error distribution. The proposed algorithm could effectively reduce the NLOS error, while the proposed algorithm needs the prior knowledge of the statistical characteristics of the system's standard measurement noise. Wann and Lin [9] proposes a method to fuse two different types of signals combining Bayes rules and fuzzy logic based on [8], which further improves localization accuracy.

Other researchers introduce weighted factor to mitigate the adverse effects from NLOS distance measurements on localization. In order to increase the robustness of the algorithm for NLOS error, the researchers give greater weight to less affected distance measurement and give less weight to greater affected distance measurement. Chan and $\mathrm{Ho}[10]$ presents the weighted linear least-squares estimation algorithm based on the Taylor series expansion. Although the algorithm has been used widely, it requires statistical information to determine the weights. Chen [11] proposes a residual weighting algorithm to mitigate the NLOS error. The proposed approach utilizes the sum of squared residuals obtained from least squares estimation as the indicator of the accuracy. Then they apply least squares approximation method and compute the estimated location. The method need not identify the distance measurement signal. Kirubarajan and Bar-Shalom [12] proposes a probabilistic data association with different probabilities to weight each distance measurement. The proposed algorithm could be effective for target tracking and is useful for a wide variety of real tracking problem.

In addition to the methods mentioned above, Wang et al. [13] presents a robust mobile target localization approach. The proposed algorithm utilizes the probability density function (PDF) estimator to identify the NLOS condition, then an improved biased Kalman filter (KF) algorithm is used to mitigate the NLOS error. The proposed algorithm needs the less prior information about communication environments, while has a good location performance even in severe NLOS situations. Mazuelas [14] utilizes known parameters and distributions of the NLOS error, computing the NLOS error from the distance measurements to effectively correct the measurements from NLOS propagation. The proposed algorithm could effectively correct the measurements from
NLOS propagation while need the prior NLOS measurement. Chan et al. [5] proposes to utilize the residual test to identify the LOS base station, so the localization can proceed with the LOS base station. In [15], a linear programming approach is introduced to mitigate the NLOS error. By using the geometry of the base station, a constrained nonlinear optimization approach is presented to solve the NLOS localization [16]. Wang et al. [17] treats localization in the LOS environment as unconstrained optimization problems. Therefore the NLOS situation localization problem is transformed into an optimization problem to solve. In [18], an NLOS localization algorithm based on semi-definite programming technique is presented, and the localization problem has been approximated by a convex optimization problem. Chen and co-author [19] proposes the interacting multiple model approach with the KF technique to interact the LOS distance measurement and the NLOS distance measurement. However, the algorithm needs to get an NLOS parameters as a priori information. In [20], a geometry-constrained location estimation (GLE) based on the two step least squares algorithm is proposed. The proposed algorithm possesses low complexity from the two-step LS algorithm and is suitable for the NLOS localization at the same time. Abumansoor et al. [21] propose a cooperative localization algorithm to mitigate the NLOS error. Yu and Dutkiewicz [22] proposes the high-frequency distance measurement data processing in base station localization. The proposed algorithm could significantly improve position accuracy. An NLOS identification and probability generation algorithm is proposed by Hammes and Zoubir [23]. In this method, the M-estimate based robust KF is used to reduce the NLOS effect and the algorithm yields positioning accuracy similar to the EKF in the LOS environments and even significantly outperforms the REKF in the NLOS environments. Lin et al. [24] proposes a NLOS identification method to identify LOS measurement and NLOS measurement by using the statistics of measurements from the $\mathrm{MN}$, but the algorithm focuses on the cellular network. Guvenc and Chong [1] presents the linear least square algorithm based on fixed $\mathrm{BN}$. The disadvantage is that the localization accuracy will decline if the $\mathrm{MN}$ is far away from the $\mathrm{BN}$ without changing the $\mathrm{BN}$ deployment environment. Hence, localization accuracy is influenced by the selection of BN. Cheng et al. [25] proposes a likelihood matrix correction based mixed Kalman and $\mathrm{H}$-infinity filter method. They propose the likelihood matrix based correction to correct the measurements, then using the mixed Kalman and $\mathrm{H}$-infinity Filter method to improve the range measurement. This method does not need the prior information about the statistical properties of the NLOS errors. But the computational complexity of the method is high. Wang and Zekavat [26] proposes an omnidirectional MN localization technique in NLOS scenarios. They proposed an algorithm to identify if a $\mathrm{MN}$ is in the LOS of multiple $\mathrm{BN}$ or not. The method 
enables $\mathrm{BN}$ to determine and localize their shared reflection points, and to localize NLOS MN.

The proposed algorithms can be used in outdoor environments in references [8,9,11,12,16,17,19,20,22-24]. The proposed methods of references $[10,13,15,18,25]$ focus on the indoor localization.

In this paper, we propose the voting weighted association reference (VWAR) algorithm and voting weighted association least (VWAL) algorithm. We firstly use data preprocessing method of measurements based on voting selection mechanisms and preserve the reliable data. Secondly, a weight value generation algorithm is proposed. The weight values of each distance measurements can be reasonably generated by the proposed algorithm. Probability data association algorithm is used to weight these distance measurements. Then, we use KF algorithm to filter the weighted distance data. Finally, we employ reference linear least square algorithm based on the selection of reference nodes to estimate the location of $\mathrm{MN}$.

The main contribution of this paper is given as follows:

(1) For the NLOS measurements, the vote selection mechanisms are proposed to filter the distance measurements. Then we propose the probabilistic data association method to mitigate the NLOS error.

(2) The proposed algorithm does not assume any statistical knowledge of the NLOS error. Therefore it is independent of the physical measurement ways.

The rest of the paper is organized as follows: In Sect. 2, we introduce the architecture of the localization algorithm. Section 3 introduces our proposed strategy in detail. In Sect. 4, some simulation and experiment results are presented. The conclusions are given in Sect. 5 .

\section{The proposed algorithm architecture}

According to whether or not the NLOS error parameters are known, NLOS localization algorithm can be divided into two categories: localization algorithms with known parameters and localization algorithms with unknown parameters. If we can accurately get the NLOS error distribution model and its parameters as prior knowledge, we can mitigate the NLOS error and achieve higher localization performance. But the NLOS propagation model in the actual environment is not consistent with prior knowledge, which decrease the localization accuracy obviously. The unknown parameters localization algorithm does not need to obtain a NLOS distribution model and its parameters as prior knowledge, so this kind of algorithm will not have great influence when the NLOS propagation environments change. The deployment environment of wireless sensor networks is complicated, especially in NLOS propagation environment, thus the unknown parameters localization algorithm has higher research value and practical significance. The proposed algorithm is an unknown parameters localization algorithm and it can guarantee higher localization accuracy in different NLOS propagation environments.

Figure 1 shows the architecture of the proposed algorithm. The proposed algorithm is referenced from the high-frequency distance measurement data processing in base station localization. Ten distance measurements can be acquired by each beacon node in each localization process. In which, $\mathbf{r}_{i}(t), i=1,2, \ldots, N$ is original distance measurement vector of the $i$ th beacon node at time $t ; \overline{\mathbf{r}}_{i}(t)$ is the distance vector composed of the reserved data filtered through voting selection method which is initially the distance measurements of each beacon node; $N_{v}(t)$ is the number of the distance measurement through voting; $\hat{\boldsymbol{\theta}}_{i}, \mathbf{P}_{i}$
Fig. 1 Architecture of the proposed localization algorithm

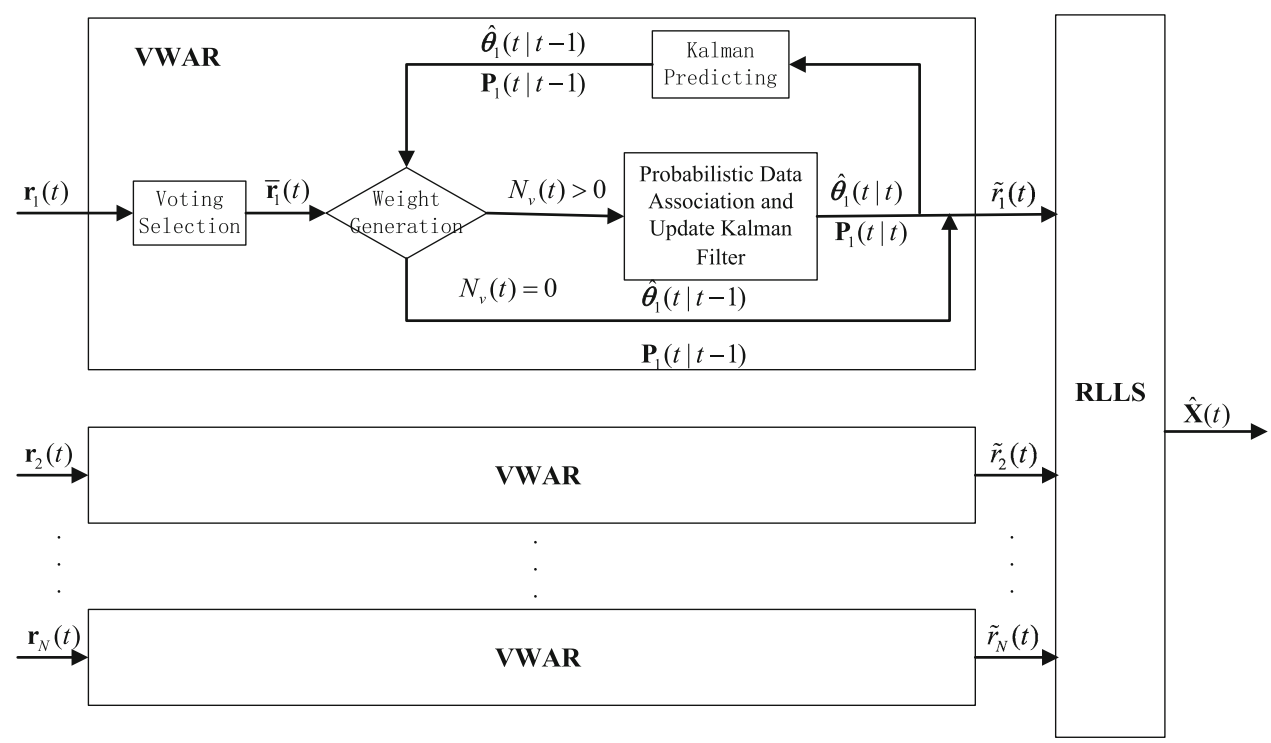


are KF parameter; $\tilde{r}_{i}(t)$ is the distance by using associating probabilistic data association and $\mathrm{KF} ; \hat{\mathbf{X}}(t)$ is the location vector of the MN through calculating. The proposed algorithm is described as follows:

(1) Voting selection mechanisms: Selecting and processing the initial distance measurements of each $\mathrm{BN}$ by voting selection mechanisms, then we reserve the reliable distance measurements to form vector $\overline{\mathbf{r}}_{i}(t)$.

(2) Weight generation: We use KF algorithm to predict state vectors, and utilize Bayes theorem to work out the weights corresponding to all the distance values of distance vector $\overline{\mathbf{r}}_{i}(t)$.

(3) Probabilistic data association and update KF: The weight values of each distance measurement in $\overline{\mathbf{r}}_{i}(t)$ can be generated by the proposed algorithm. Then probability data association algorithm is used to weight these distance measurements. Using $\mathrm{KF}$ algorithm to filter the weighted distance data, we can obtain the filtered distance measurement $\tilde{r}_{i}(t)$ in real time.

(4) Localization: Using the filtered distance measurement $\tilde{r}_{i}(t)$ from step (3) and linear least square algorithm based on the selection of BN to complete the localization calculation.

\section{NLOS localization based on voting selection mechanisms}

\subsection{System model}

In order to locate the $\mathrm{MN}, N \mathrm{BN}$ are considered in this paper. The coordinate of the $\mathrm{BN}$ are represented as $\left[x_{i}, y_{i}\right]^{\mathbf{T}}, i=$ $1,2, \ldots, N$; the coordinate of the mobile node at time $t$ is represented as $[x(t), y(t)]^{T}$; the measured distance can be acquired by multiplying the arrival time and the speed of signal. The real distance between the $\mathrm{MN}$ and the $i$ th beacon node at the time $t$ is :

$d_{i}(t)=\sqrt{\left(x(t)-x_{i}\right)^{2}+\left(y(t)-y_{i}\right)^{2}}$

In the LOS environment, the measured distance between the mobile node and the $i$ th beacon node at the time $t$ is:

$r_{i}(t)=d_{i}(t)+e_{L O S}$

where $e_{L O S}$ is the measurement noise error [27], which is modeled as zero-mean white Gaussian and standard deviation is $\sigma_{g}$.

In the NLOS propagation environment, the measured distance at the time $t$ is modeled as:

$r_{i}(t)=d_{i}(t)+e_{L O S}+e_{N L O S}$ where $e_{N L O S}$ is the NLOS error and independent with $e_{L O S}$. In different kinds of environments, $e_{N L O S}$ could obey Exponential distribution, Gaussian distribution or Uniform distribution [28].

\subsection{Measurement data preprocessing method based on voting selection mechanisms}

The differences of the mean values and standard deviations between LOS and NLOS distance measurements are used in the measurement data preprocessing method based on voting selection mechanisms. In order to provide the reliable remained data for following data processing, the unreliable part is eliminated by reasonable filtrating of the initial distance measurement.

The precondition of using the proposed algorithm is that it is necessary to assume the sampling frequency of distance measurement is larger than 1 , which is $10 \mathrm{~Hz}$, and the localization frequency is set as $1 \mathrm{~Hz}$ in this paper. The idea is referenced from the high-frequency distance measurement data processing in base station localization [22].

Considering the temporal interval $[0, K]$ of the whole localization process, $L$ distance measurements are executed by beacon node $i$. $L$ distance measurements are shown as follows:

$\varpi=\left\{r_{k} \mid k=1,2, \ldots, L\right\}$

Then, the set $\varpi$ is divided as $L-Z+1$ interlaced subsets according to the sampling time sequence. Each subset contains $Z$ distance measurements, concluded within set $\psi$ :

$\psi=\left\{\mathbf{R}_{j} \mid j=1,2, \ldots, L-Z+1\right\}$

where the parameter $Z$ needs to consider the set $\boldsymbol{R}_{j}$ contains enough samples for analyzing the statistical property. In the corresponding temporal interval with $Z$ distance measurements in $\boldsymbol{R}_{j}$, the moving distance value of MN should not be too large for the robustness of the statistical analysis. Therefore the $Z$ is set to be 10 , which is accordant with the distance measurement frequency.

We sort the distance measurements in each $\boldsymbol{R}_{j}$ by value. Meanwhile, indexing label of each measurement is remained which is expressed as follows:

$\mathbf{R}_{j}^{a}=\left\{r^{1}, r^{2}, \ldots, r^{Z}\right\}, j=1,2, \ldots, L-Z+1$

where $\mathbf{R}_{j}^{a}$ is the sorted measurement, $r^{1}, r^{2}, \ldots, r^{Z} \in \varpi$, and $r^{1}<r^{2}<\cdots<r^{Z}$.

Selecting the smallest $l$ measurements in $\mathbf{R}_{j}^{a}$, we calculate the mean value $\hat{\mu}_{j(l)}$ and standard deviation $\hat{\sigma}_{j(l)}$. The mean sequence $\mu_{j}^{a}$ and standard deviation sequence $\sigma_{j}^{a}$ of $\mathbf{R}_{j}^{a}$ can 
be obtained:

$$
\begin{aligned}
\mu_{j}^{a} & =\left\{\hat{\mu}_{j(2)}, \hat{\mu}_{j(3)}, \ldots, \hat{\mu}_{j(Z)}\right\} \\
\sigma_{j}^{a} & =\left\{\hat{\sigma}_{j(2)}, \hat{\sigma}_{j(3)}, \cdots, \hat{\sigma}_{j(Z)}\right\}
\end{aligned}
$$

Comparing each estimated standard deviation in standard deviation sequence $\sigma_{j}^{a}$ in each $\boldsymbol{R}_{j}$, we find the closest value of estimated standard deviation $\hat{\sigma}_{m}$ to the measurement error standard deviation $\sigma_{g}$. The distance measurement, with which the estimated standard deviation is calculated, can be selected to get one vote. Finally, the voting weight of each distance measurement can be acquired:

$\alpha(k)=\frac{c_{k}}{c_{Z}}, k=1,2, \ldots, L$

where $\alpha(k)$ is the voting weight value of distance measurement $r_{k}, c_{k}$ is the final vote of $r_{k}, c_{Z}$ is the probable maximum vote of $r_{k}$, namely, $c_{Z}=Z$. We treat the distance measurements with voting weight value $\alpha(k) \geq 0.5$ as reliable data, and that with voting weight value $\alpha(k)<0.5$ as unreliable data. Then we remain reliable data and eliminate unreliable data in following processing.

In this paper, the distance measurement frequency has been set as $10 \mathrm{~Hz}$ and the localization frequency is $1 \mathrm{~Hz}$ in the proposed algorithm. Then in each localization process, 10 distance measurements can be acquired by each beacon node. Using all of the distance measurements to form an initial distance vector $\mathbf{r}(t)$. By voting selection, reserve the reliable distance measurements as vector $\overline{\mathbf{r}}(t)$ for following calculation.

When the beacon node is under the LOS or NLOS situation, all the measurements in $\boldsymbol{R}_{j}$ are more probable to be LOS measurements or NLOS measurements. When under LOS situation, the measurements in $\boldsymbol{R}_{j}$ can be mostly remained for following calculating through the voting selection. When under NLOS situation, the NLOS standard deviation is usually larger than the LOS ones, the NLOS measurements with small errors can be selected for calculating in order to compare with the measure standard deviation.

\subsection{Probabilistic data association algorithm based on voting selection mechanisms}

Using measured data preprocessing method based on voting selection mechanisms, distance vector $\overline{\mathbf{r}}(t)$ which contains reliable measured data can be obtained. After that, we process the distance in vector $\overrightarrow{\mathbf{r}}(t)$ with weight value generation and probability data association. The result is filtered through $\mathrm{KF}$. Since the number of BN is $N$, the number of KFs is also $N$ to filter all data from BN. The state equation corresponding with the beacon node is defined as follows [29]:
$\theta(t)=\mathbf{A} \theta(t-1)+\mathbf{G} v(t-1)$

where $\boldsymbol{\theta}(t)$ is the state vector of MN relative to $i$ th beacon node $\boldsymbol{\theta}(t)=[r(t), v(t)]^{\mathbf{T}}$; stochastic process $v(t-1)=$ $\left[v_{r_{t-1}}, v_{v_{t-1}}\right]^{\mathbf{T}}$ is white Gaussian noise; matrix $\mathbf{A}$ and $\mathbf{G}$ are defined as:

$\mathbf{A}=\left[\begin{array}{cc}1 & \Delta k \\ 0 & 1\end{array}\right], \quad \mathbf{G}=\left[\begin{array}{c}0.5 \Delta k^{2} \\ \Delta k\end{array}\right]$

where $\Delta k$ is the period of localization.

The measurement equation of system is:

$r_{n}(t)=\mathbf{H} \boldsymbol{\theta}(t)+v_{n}(t), n=1,2, \ldots, N_{v}(t)$

where $\mathbf{H}$ is observation matrix, defined as $\mathbf{H}=[1,0]$; the vector $v_{n}(t)$ is random variable caused by measurement noise of sensors, follows Gaussian distribution whose mean value is 0 and standard deviation is $\sigma_{g} ; N_{v}(t)$ is the dimension of $\overline{\mathbf{r}}(t)$.

The time update equation of KF can be expressed as follows:

$\hat{\boldsymbol{\theta}}(t \mid t-1)=\mathbf{A} \hat{\boldsymbol{\theta}}(t-1 \mid t-1)$

$\mathbf{P}(t \mid t-1)=\mathbf{A P}(t-1 \mid t-1) \mathbf{A}^{\mathbf{T}}+\mathbf{G Q G}^{\mathbf{T}}$

where $\mathbf{Q}$ is the variance of system processing noise.

Forming the reference of weight generation, and the residual variable is generated as:

$$
\begin{aligned}
\hat{r}(t \mid t-1) & =\mathbf{H} \hat{\boldsymbol{\theta}}(t \mid t-1) \\
\gamma_{n}(t) & =\bar{r}_{n}(t)-\hat{r}(t \mid t-1), n=1,2, \ldots, N_{v}(t)
\end{aligned}
$$

where $\hat{r}(t \mid t-1)$ is the reference of weight generation; $\bar{r}_{n}(t)$ is the $n$th distance measurement in $\overline{\mathbf{r}}(t) ; \gamma_{n}(t)$ is the residual error caused by $\bar{r}_{n}(t)$.

Equation (17) can be derived from Eqs. (12) and (16):

$\gamma_{n}(t) \sim N\left(0, T_{n}(t)\right), n=1,2, \ldots, N_{v}(t)$

where

$T_{n}(t)=\mathbf{H P}(t \mid t-1) \mathbf{H}^{\mathbf{T}}+\sigma_{g}^{2}, n=1,2, \ldots, N_{v}(t)$

In each localization process, only one distance measurement $\bar{r}_{j}(t)$ is deemed as the highest accurate distance measurement data. Other data in $\overline{\mathbf{r}}(t)$ can be treated as less reliable clutter data since the existence of LOS or NLOS errors, which follows Uniform distribution in confidence interval. In order to explain the weight generation algorithm, we define as follows:

$\delta_{j}(t):\left\{\bar{r}_{j}(t)\right.$ is the highest accurate distance measurement,

$\left.j=1,2, \ldots, N_{v}(t)\right\}$ 
The associated probability can be obtained [30]:

$w_{j}(t)=\operatorname{Pr}\left\{\delta_{j}(t) \mid \mathbf{R}^{t}\right\}, j=1,2, \ldots, N_{v}(t)$

Equation (20) can be inferred as:

$w_{j}(t)=\operatorname{Pr}\left\{\delta_{j}(t) \mid \mathbf{R}(t), N_{v}(t), \mathbf{R}^{t-1}\right\}, j=1,2, \ldots, N_{v}(t)$

where $\mathbf{R}(t)=\left\{\bar{r}_{j}(t)\right\}_{j=1}^{N_{v}(t)} ; \mathbf{R}^{t}$ is the accumulation set of distance measurements, namely, $\mathbf{R}^{t}=\{\mathbf{R}(i)\}_{i=1}^{t}$.

Since the residual error variables $\gamma_{j}(t), j=1,2, \ldots$, $N_{v}(t)$ are mutually independent, applying Bayes theorem, Eq. (21) can be rewritten as:

$$
\begin{aligned}
w_{j}(t)= & \frac{1}{c} f\left(\mathbf{R}(t) \mid \delta_{j}(t), N_{v}(t), \mathbf{R}^{t-1}\right) \\
& \times \operatorname{Pr}\left\{\delta_{j}(t) \mid N_{v}(t), \mathbf{R}^{t-1}\right\}, \quad j=1,2, \ldots, N_{v}(t)
\end{aligned}
$$

where, $c$ is normalization constant; $f(\cdot)$ is the joint density function for each distance measurement under the condition of $\delta_{j}(t), N_{v}(t)$ and $\mathbf{R}^{t-1}$. For $\delta_{j}(t), f(\cdot)$ is the product of the accurate data followed by Gaussian PDF and the clutter data in confidential interval followed by Uniform distribution probability density function.

The PDF of accurate data is the Gaussian PDF whose mean value is $\hat{r}(t \mid t-1)$ :

$f\left(\bar{r}_{j}(t) \mid \delta_{j}(t), N_{v}(t), \mathbf{R}^{t-1}\right)=N\left(\bar{r}_{j}(t) ; \hat{r}(t \mid t-1), T_{j}(t)\right)$

It can be inferred from Eq. (23):

$f\left(\bar{r}_{j}(t) \mid \delta_{j}(t), N_{v}(t), \mathbf{R}^{\mathrm{t}-1}\right)=\frac{\exp \left\{-0.5 \gamma_{j}^{2}(t) / T_{j}(t)\right\}}{\sqrt{2 \pi\left|T_{j}(t)\right|}}$

According to the Eq. (23), $f\left(\mathbf{R}(t) \mid \delta_{j}(t), N_{v}(t), \mathbf{R}^{t-1}\right)$ in Eq. (22) can be expressed as:

$$
\begin{aligned}
f & \left(\mathbf{R}(t) \mid \delta_{j}(t), N_{v}(t), \mathbf{R}^{t-1}\right) \\
& =\prod_{i=1, i \neq j}^{N_{v}(t)} \frac{N\left(\gamma_{j}(t) ; 0, T_{j}(t)\right)}{\lambda_{i}(t)}, j=1, \ldots, N_{v}(t)
\end{aligned}
$$

where $\lambda_{i}(t)$ is confidence region and defined as:

$$
\lambda_{i}(t)=\varepsilon \pi \sqrt{\left|T_{j}(t)\right|}
$$

where $\varepsilon$ is chosen as 1 .
Therefore, in Eq. (25), accurate data is deemed as following Gaussian distribution whose mean value is $\hat{r}(t \mid t-1)$. On the other hand, the clutter data is deemed is modeled by a uniform probability distribution in the confidence interval of $\lambda_{i}(t)$. The less the distance measurement $\bar{r}_{j}(t)$ differs from weight generation standard $\hat{r}(t \mid t-1)$, the less the value of residual error $\gamma_{j}(t)$ is. Hence in order to have more effect in localization calculation with $\bar{r}_{j}(t)$, it is probable to generate a large weight value $w_{j}^{\prime}(t)$.

The prior probability of Eq. (22) is:

$\operatorname{Pr}\left\{\delta_{j}(t) \mid N_{v}(t), \mathbf{R}^{t-1}\right\}=\frac{1}{N_{v}(t)}, j=1,2, \ldots, N_{v}(t)$

Multiplying Eq. (25) by (27) leads to:

$w_{j}^{\prime}(t)=\frac{N\left(\gamma_{j}(t) ; 0, T_{j}(t)\right)}{N_{v}(t)} \prod_{i=1, i \neq j}^{N_{v}(t)} \frac{1}{\lambda_{i}(t)}$

Normalizing the Eq. (28), the weight value $\bar{r}_{j}(t)$ is processed as:

$$
w_{j}(t)=\frac{w_{j}^{\prime}(t)}{\sum_{i=1}^{N_{v}(t)} w_{j}^{\prime}(t)}, \forall j=1,2, \ldots, N_{v}(t)
$$

Then weighting each distance measurement in $\overline{\mathbf{r}}(t)$, and combining with KF algorithm. For calculating Kalman gain, firstly define update variance as [12]:

$T(t)=\mathbf{H P}(t \mid t-1) \mathbf{H}^{\mathbf{T}}+\sigma_{g}^{2}$

And the Kalman gain is:

$\mathbf{K}(t)=\mathbf{P}(t \mid t-1) \mathbf{H}^{\mathbf{T}} T^{-1}(t)$

The final state estimated value is obtained from using weighted distance measurement as:

$\hat{\boldsymbol{\theta}}(t \mid t)=\hat{\boldsymbol{\theta}}(t \mid t-1)+\mathbf{K}(t) \sum_{j=1}^{N_{v}(t)} w_{j}(t) \bar{r}_{j}(t)$

The covariance updating equation can be expressed as:

$\mathbf{P}(t \mid t)=\mathbf{P}_{L}(t \mid t)+\overline{\mathbf{P}}(t)$

where $\mathbf{P}_{L}(t \mid t)$ is the posteriori covariance matrix of standard $\mathrm{KF}$ algorithm, and

$$
\begin{aligned}
\mathbf{P}_{L}(t \mid t) & =\left(\mathbf{E}_{2}-\mathbf{K}(t) \mathbf{H}\right) \mathbf{P}(t \mid t-1) \\
\overline{\mathbf{P}}(t) & =\mathbf{K}(t)\left[\sum_{j=1}^{N_{v}(t)} w_{j}(t) \gamma_{j}^{2}(t)-\gamma^{2}(t)\right] \mathbf{K}^{\mathbf{T}}(t)
\end{aligned}
$$


where

$\gamma(t)=\sum_{j=1}^{N_{v}(t)} w_{j}(t) \gamma_{j}(t)$

Above is under the situation of $N_{v}(t)>0$, which is relatively normal. However, the situation of $N_{v}(t)=0$ is also probable. When $N_{v}(t)=0$, it is shown as follows:

$\hat{\boldsymbol{\theta}}(t \mid t)=\hat{\boldsymbol{\theta}}(t \mid t-1)$

$\mathbf{P}(t \mid t)=\mathbf{P}(t \mid t-1)$

After calculating the posteriori state estimation vector $\hat{\boldsymbol{\theta}}(t \mid t)$, the distance value used in localization calculation can be obtained from:

$\tilde{r}(t)=[1,0] \hat{\boldsymbol{\theta}}(t \mid t)$

\subsection{Linear least square algorithm based on the selection of beacon node}

We assume that the coordinates of $\mathrm{BN}$ are $\left(x_{1}, y_{1}\right),\left(x_{2}, y_{2}\right)$, $\ldots,\left(x_{N}, y_{N}\right)$, the coordinate of the $\mathrm{MN}$ is $(x(t), y(t))$. According to the processed distances $\tilde{r}_{1}(t), \tilde{r}_{2}(t), \ldots, \tilde{r}_{N}(t)$ in the 3.2 and the coordinates of both $\mathrm{BN}$ and the $\mathrm{MN}$, the following linear equation can be concluded:

$\mathbf{D Y}=\frac{1}{2} \mathbf{B}$

where

$\begin{aligned} & \mathbf{D}= {\left[\begin{array}{cc}\left(x_{2}-x_{1}\right) & \left(y_{2}-y_{1}\right) \\ \left(x_{3}-x_{1}\right) & \left(y_{3}-y_{1}\right) \\ \vdots & \vdots \\ \left(x_{N}-x_{1}\right) & \left(y_{N}-y_{1}\right)\end{array}\right], } \\ & \mathbf{B}=\left[\begin{array}{c}\tilde{r}_{1}^{2}-\tilde{r}_{2}^{2}-\left(x_{1}^{2}+y_{1}^{2}\right)+\left(x_{2}^{2}+y_{2}^{2}\right) \\ \tilde{r}_{1}^{2}-\tilde{r}_{3}^{2}-\left(x_{1}^{2}+y_{1}^{2}\right)+\left(x_{3}^{2}+y_{3}^{2}\right) \\ \vdots \\ \tilde{r}_{1}^{2}-\tilde{r}_{N}^{2}-\left(x_{1}^{2}+y_{1}^{2}\right)+\left(x_{N}^{2}+y_{N}^{2}\right)\end{array}\right]\end{aligned}$

By using linear least square algorithm, the coordinate of the mobile node can be achieved as:

$\mathbf{Y}=\frac{1}{2}\left(\mathbf{D}^{\mathbf{T}} \mathbf{D}\right)^{-1} \mathbf{D}^{\mathbf{T}} \mathbf{B}$

The first beacon node is selected as the reference node in Eq. (40), thus the linear least square algorithm is based on fixed reference node. The linear least square algorithm based on the selection of reference node is adopted in this paper. When establishing linear equations in Eq. (40), the beacon node relevant to the smallest distance measurement is always to be chosen as reference node, which is shown as:

$\mathbf{Y}_{\mathbf{b r}}=\arg \min _{i}\left\{\tilde{r}_{i}\right\}, i=1,2, \ldots, N$

Being similar with standard linear least square algorithm, the one based on the selection of reference node is one of LOS localization algorithms. From Eq. (42), the distance measurement from beacon node directly influences the selection of reference node. In the NLOS environment, the selection of reference node is effected by inaccurate NLOS distance measurement. However, the initial distance measurement is filtrated by voting selection mechanisms in the proposed algorithm. The remained reliable distance measurement is used in the final localization calculation through processing by probability data association algorithm. Moreover, the negative influence on localization result has been eliminated or at least weakened by filtering unreliable NLOS distance measurement.

\section{Simulation and experiment results}

\subsection{Simulation results}

In this section, we analyze the performance of the proposed algorithm described in Sect. 3 through simulation and experiment. Figure 2 shows the placement of seven $\mathrm{BN}$ in the $100 \mathrm{~m} \times 100 \mathrm{~m}$ square area and one $\mathrm{MN}$ in this area. The acceleration of the $\mathrm{MN}$ is variable. We assume that the communication range of sensor node is $150 \mathrm{~m}$. The measurement noise obeys Gaussian distribution whose mean value is zero and the standard deviation $\sigma_{g}$ is $3 \mathrm{~m}$ [31]. The NLOS errors obey Exponential distribution whose mean value $\mu_{N L O S}$ $=5 \mathrm{~m}$ [32]. We compare the proposed VWAR algorithm and VWAL algorithm with the KF Linear Least Squares (KF-LLS) algorithm [29], the H-Infinity filter Linear Least Square (HH-LLS) algorithm [33] and the Particle filter Linear Least Square (PF-LLS) algorithm [34]. The simulation results of the proposed algorithm are verified through MATLAB R2010a. In each simulation case, 50 Monte Carlo runs are performed with the same parameters. The performance of the proposed algorithm is measured by Root Mean Square Error (RMSE) and Cumulative Distribution Function:

$R M S E_{t}=\sqrt{\frac{1}{M C} \sum_{j=1}^{M C}\left(x_{j}(t)-\hat{x}_{j}(t)\right)^{2}+\left(y_{j}(t)-\hat{y}_{j}(t)\right)}$

where $\left(x_{j}(t), y_{j}(t)\right),\left(\hat{x}_{j}(t), \hat{y}_{j}(t)\right)$ describes the true and the estimated position of MN at time $t$ for the $j$ th Monte Carlo run, respectively. 


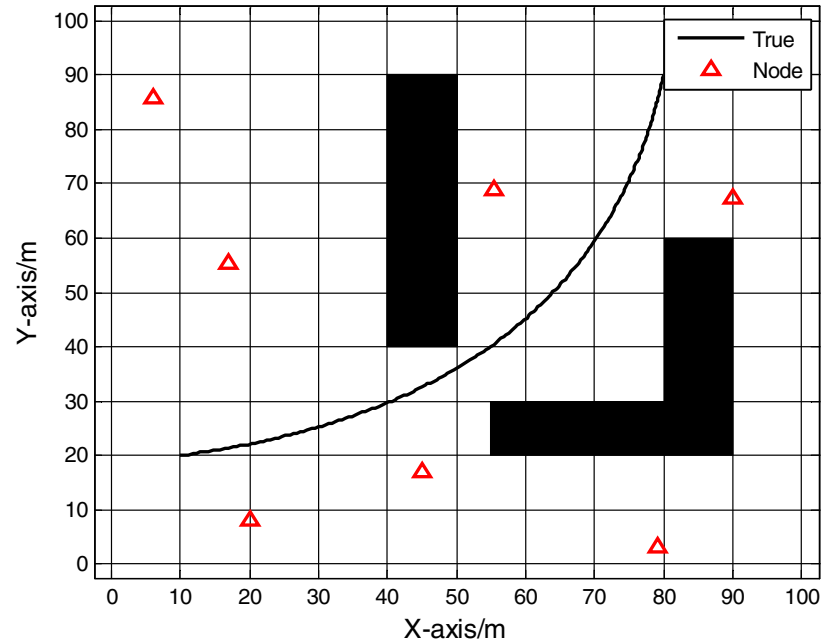

Fig. 2 The schematic diagram of the deployment environment

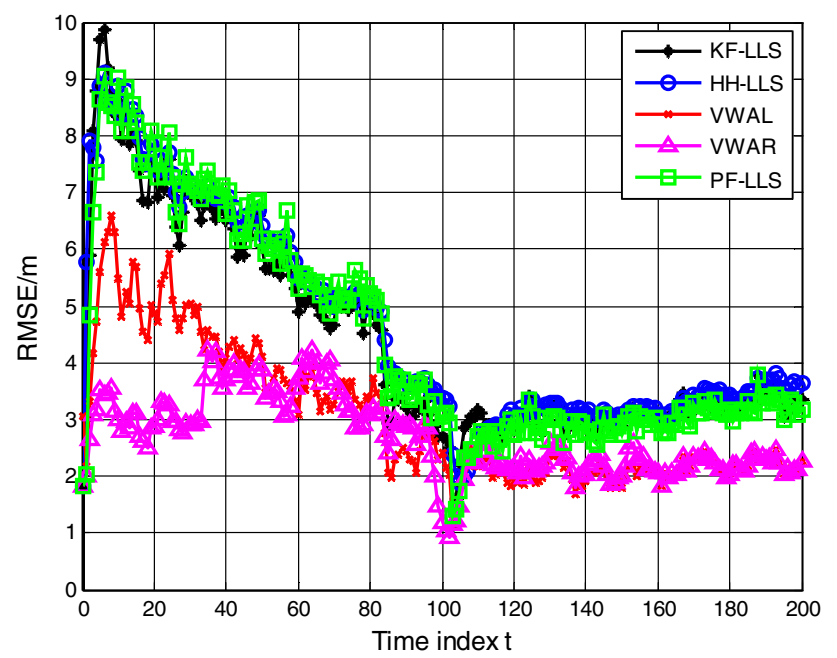

Fig. 3 Comparison of the root mean square error for five algorithms

Figure 2 shows that the deployment of the BN, obstacles and the trajectory of the MN.

Figure 3 shows the comparison of the root mean square error for five algorithms. The localization errors of KFLLS algorithm, HH-LLS algorithm and PF-LLS algorithm are relatively larger than the proposed algorithms. KF-LLS algorithm, HH-LLS algorithm and PF-LLS algorithm are sensitive to NLOS error, these localization methods are easier to be affected by the NLOS error. In contrast, the localization performance of the VWAL algorithm and VWAR algorithm are stable and the localization accuracy improve greatly. It can be seen that VWAR algorithm and VWAL algorithm have strong inhibition for NLOS error, and VWAR algorithm has the highest localization accuracy.

Figure 4 shows the cumulative distribution function of localization error for the five algorithms. It can be observed that the 90-percentile of the KF-LLS algorithm is less than 7.1

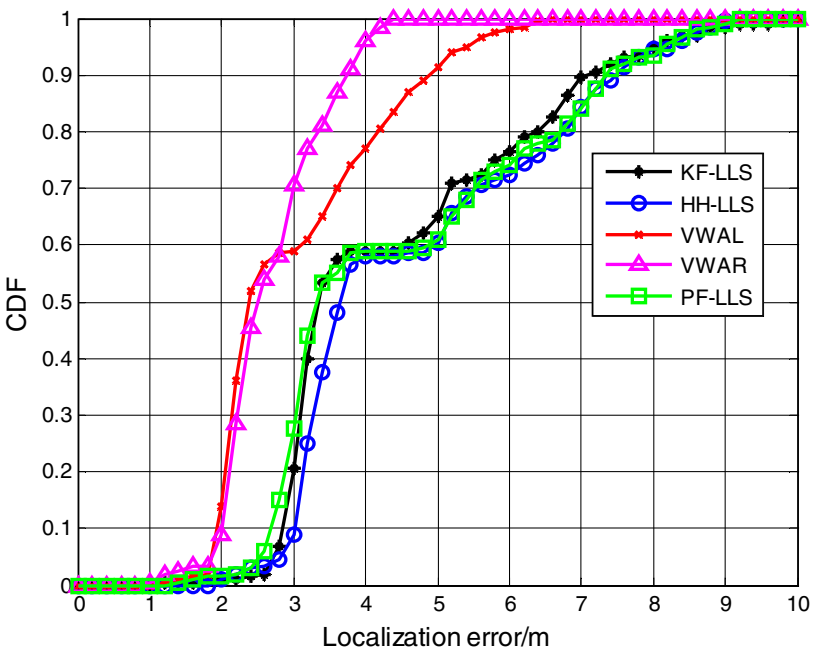

Fig. 4 The cumulative distribution function of localization error

$\mathrm{m}$, the 90-percentile of the PF-LLS algorithm is less than 7.4 $m$ and the 90-percentile of the HH-LLS algorithm is less than $7.5 \mathrm{~m}$. The percentiles of the localization error of the VWAR algorithm is $90 \%$ less than $3.7 \mathrm{~m}$ and the percentiles of the localization error of the VWAL algorithm is $90 \%$ less than $4.9 \mathrm{~m}$. Therefore, the localization performance of the VWAR algorithm and the VWAL algorithms are far superior to PFLLS algorithm, KF-LLS algorithm and HH-LLS algorithm, and the VWAR algorithm has slightly higher localization accuracy than the VWAL algorithm.

In the following section, we evaluate the performance of our proposed algorithm when the NLOS errors obey the Uniform distribution, the Gaussian distribution and the Exponential distribution.

\subsubsection{The NLOS errors obey uniform distribution}

When the NLOS errors obey a Uniform distribution, the minimum and maximum values of parameters of Uniform distribution are 1 and $U_{\max }$. Figure 5 shows the relationship between $U_{\max }$ and root mean square error. We observe that the localization errors of the five algorithms increase as the $U_{\max }$ increases. When the $U_{\max }=2$, the VWAR algorithm has higher localization accuracy than HH-LLS, PF-LLS KF-LLS and VWAL algorithms about 32.35, 22.30, 14.81 and $11.54 \%$, respectively. When the $U_{\max }=14$, the VWAR algorithm is increased by $42.86,41.18,40.87$ and $6.85 \%$. Thus by increasing $U_{\max }$, the localization accuracy of the HH-LLS, PF-LLS and KF-LLS algorithms decrease significantly. Therefore the localization performance of the proposed algorithms is stable and the VWAR algorithm has the higher localization accuracy than the VWAL algorithm.

Figure 6 illustrates the measurement noise standard deviation versus RMSE. We can observe that VWAR algorithm has 


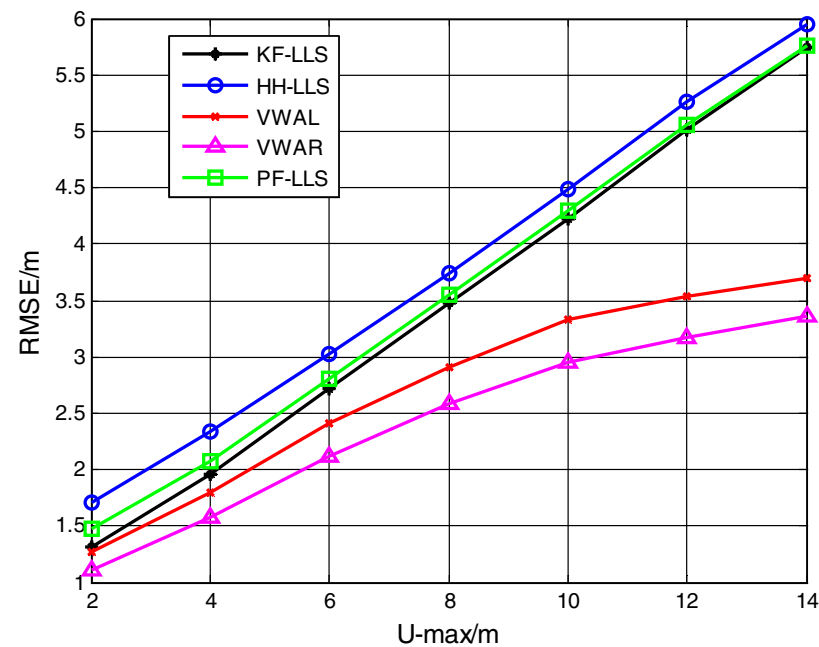

Fig. 5 The U-max versus root mean square error

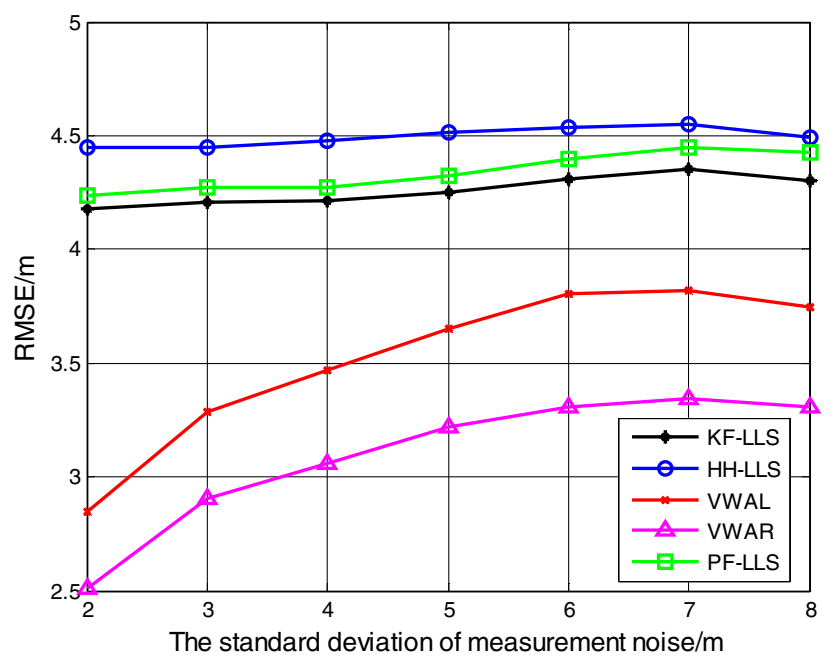

Fig. 6 The standard deviation of measurement noise versus root mean square error

the highest localization accuracy and the localization error does not exceed the other algorithms localization errors.

\subsubsection{The NLOS errors obey Gaussian distribution}

When the NLOS errors obey a Gaussian distribution, the performance of each algorithm is discussed in two cases. Figure 7 gives the mean of NLOS error versus RMSE. With the increase of the mean of NLOS error, the localization error increases. In contrast, VWAR algorithm always has the highest localization accuracy. VWAL algorithm is slightly worse than VWAR algorithm, but much higher than the KF-LLS algorithm, HH-LLS algorithm and PF-LLS algorithm. This indicates VWAR algorithm and VWAL algorithm have weak effect by NLOS.

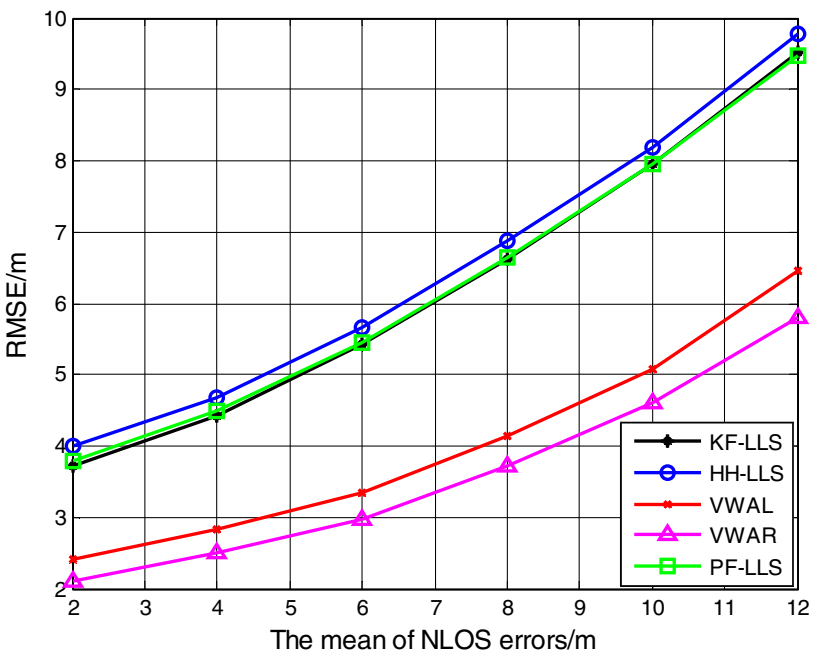

Fig. 7 The mean of NLOS errors versus root mean square error

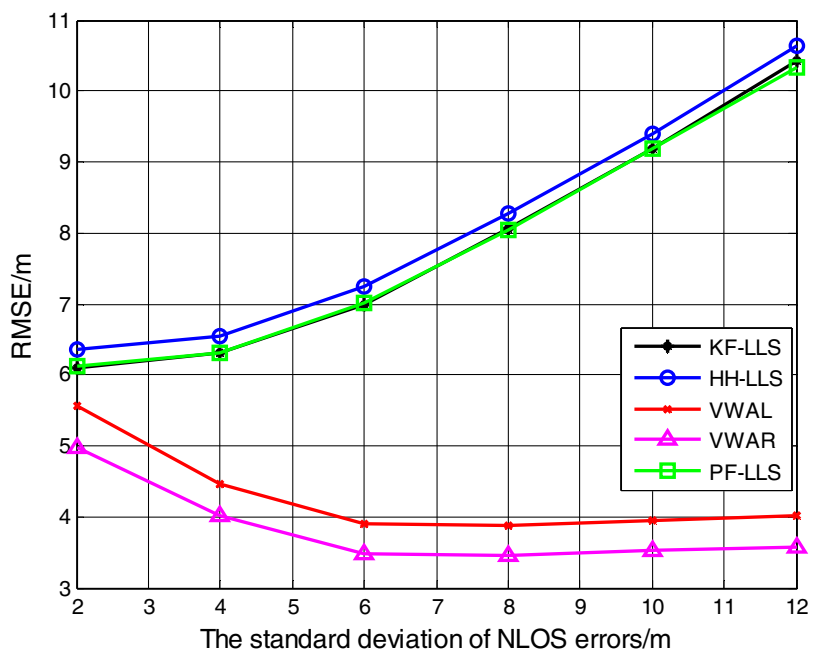

Fig. 8 The standard deviation of NLOS errors versus root mean square error

Figure 8 shows the relationship between the standard deviation of NLOS error and RMSE. It can be observed that with the increase of the standard deviation of NLOS error, the localization error of KF-LLS, HH-LLS and PF-LLS algorithms increase significantly, while the localization errors of VWAL and VWAR keep descending and finally flatten out. Therefore both VWAR and VWAL can obtain satisfactory localization effects when the standard deviations of NLOS errors are rather large.

\subsubsection{The NLOS errors obey exponential distribution}

When the NLOS errors obey an Exponential distribution, the performance of each algorithm is discussed in two cases. Figure 9 illustrates the mean of NLOS error versus RMSE. It shows all the localization accuracy increase with the mean of 


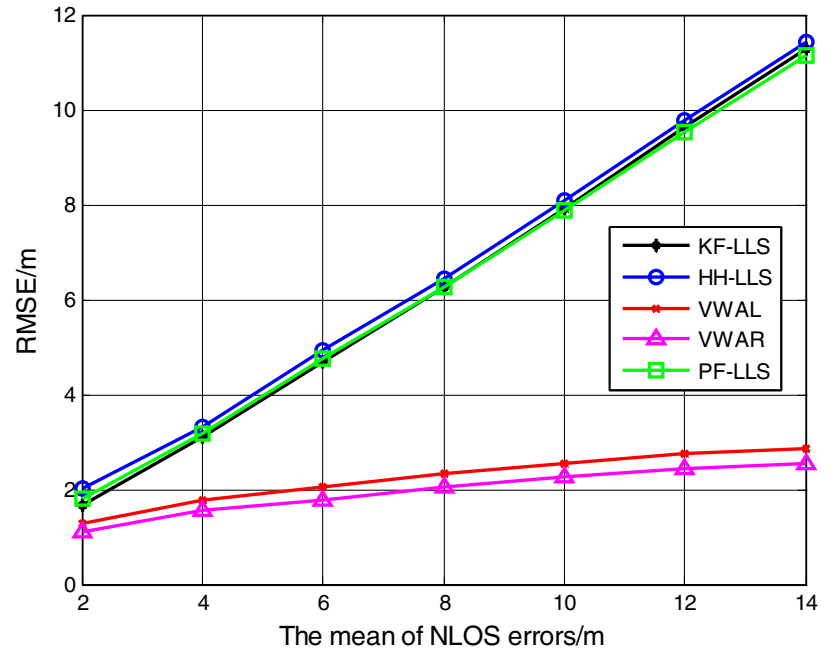

Fig. 9 The mean of NLOS errors versus root mean square error

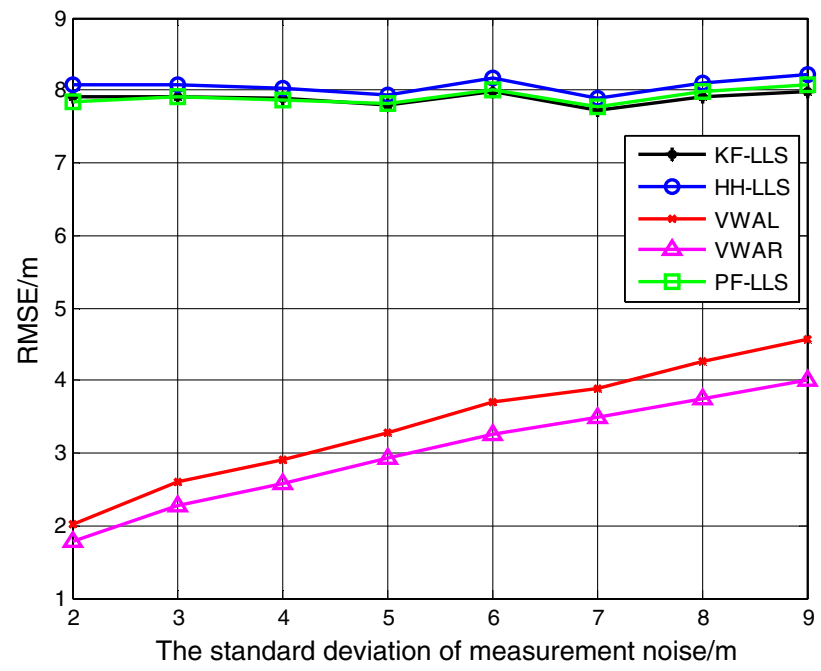

Fig. 10 The standard deviation of measurement noise versus root mean square error

NLOS decrease. When the mean of NLOS error $\mu_{N L O S}=4$ $\mathrm{m}$, the localization of the VWAR algorithm improves 46, 40, 34.55 and $14.96 \%$ when comparing with HH-LLS, PF-LLS, KF-LLS and VWAL algorithms. When the mean of NLOS error $\mu_{N L O S}=14 \mathrm{~m}$, the localization of the VWAR algorithm improves 77.84, 77.54, 77.22 and $10.56 \%$ when comparing with HH-LLS, PF-LLS, KF-LLS and VWAL algorithms. Thus it can be seen that NLOS errors have a considerable impact on the KF-LLS, PF-LLS and HH-LLS algorithms. When the mean of NLOS error increases, localization accuracy declines in an apparent way. Otherwise, the localization performance of VWAL and VWAR algorithms are relatively stable and less affected by the mean of NLOS error. The localization accuracy of VWAL algorithm and VWAR algorithm are close while the localization accuracy of VWAR algorithm stays highest among all five algorithms mentioned.

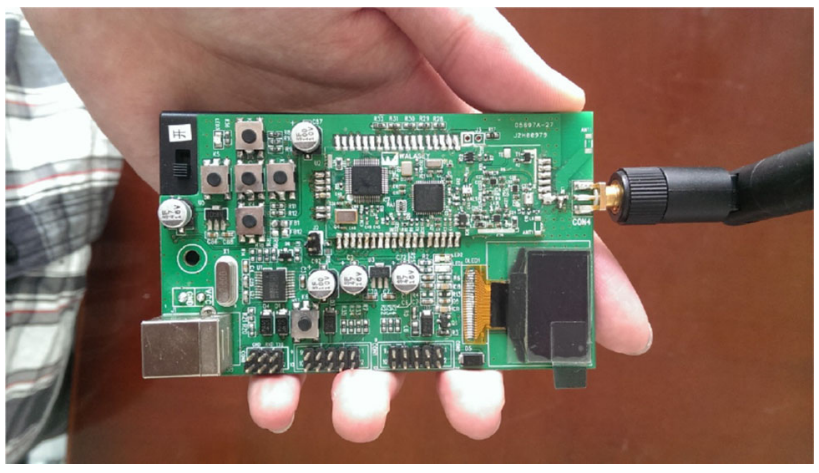

Fig. 11 The physical map of CSS node

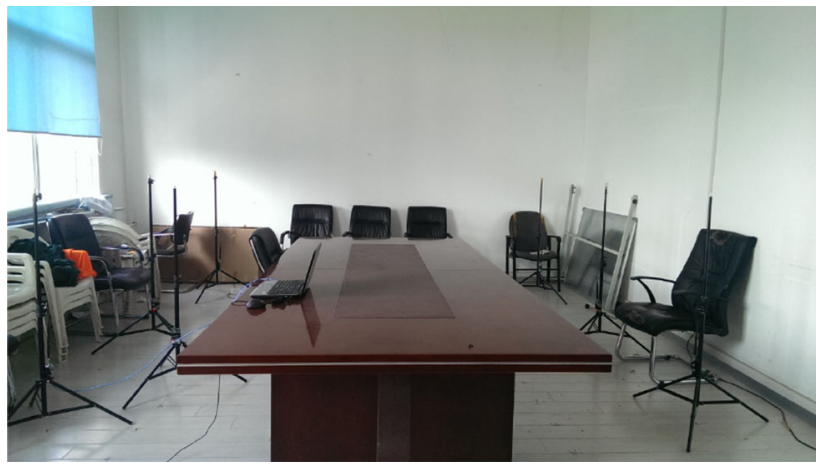

Fig. 12 The deployment diagram of the field experiment

Figure 10 shows the standard deviation of measurement noise versus root mean square error. We can observe when NLOS error obeys Exponential distribution, the localization performance of the KF-LLS algorithm, the HH-LLS algorithm and the PF-LLS algorithm are relatively stable, and less affected by the standard deviation variation of measurement noises, but the localization errors still maintain high. The localization accuracy of the VWAL algorithm and the VWAR algorithm decline with the standard deviation of measurement noise increase, but their accuracy are always much higher than the previous three algorithms.

\subsection{Experiment results}

So as to testify the localization performance of the proposed algorithm under practical environment, this paper designed field experiments to verify our methods. The experimental equipment used for field experiments is chirp spread spectrum (CSS) localization system, mainly consisting of CSS nodes. It is rather convenient to measure distance between nodes with CSS nodes. During locating experiments, BN and target node (i.e., mobile node) are established through switching working modes with CSS nodes. The physical map of CSS node is shown in Fig. 11.

Fig. 12 shows the deployment diagram of the field experiment 


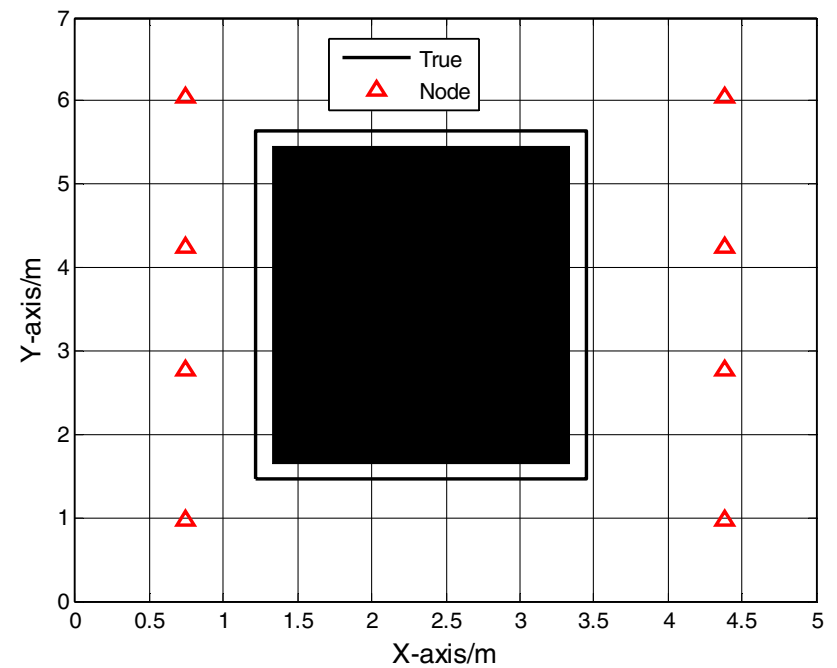

Fig. 13 The schematic diagram of the field experiment deployment environment

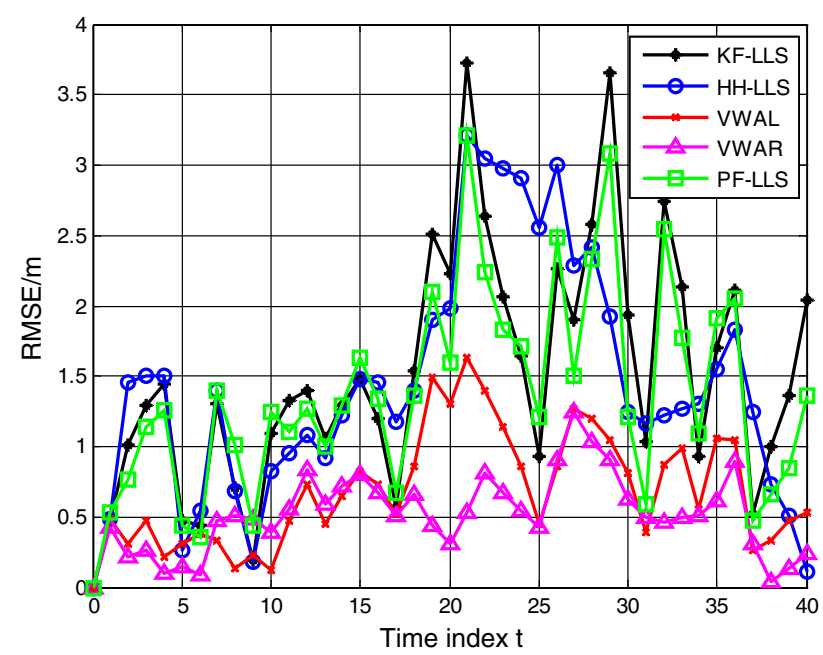

Fig. 14 Comparison of root mean square error in the field experiment

Figure 13 is the deployment environment of the field experiment.

As the figure shows, we deploy $8 \mathrm{BN}$ in a $5 \mathrm{~m} \times 7 \mathrm{~m}$ room, $\mathrm{MN}$ moves around a rectangle table in uniform velocity (long tables constitute the obstacles between nodes, which are not shown in this figure) following a rectangle trajectory. Through large quantities of distance measurement experiments, we find that under circumstances of LOS, the measurement error standard deviation of CSS node is around $0.7 \mathrm{~m}$. Considering the experimental region size, the measurement error to this extent will apparently affect the localization performance of this algorithm. Thus, the distance measurement frequency of CSS nodes is set to $20 \mathrm{~Hz}$, and 20 distance measurements are carried out at each sampling site in order to weaken the adverse impacts imposed to

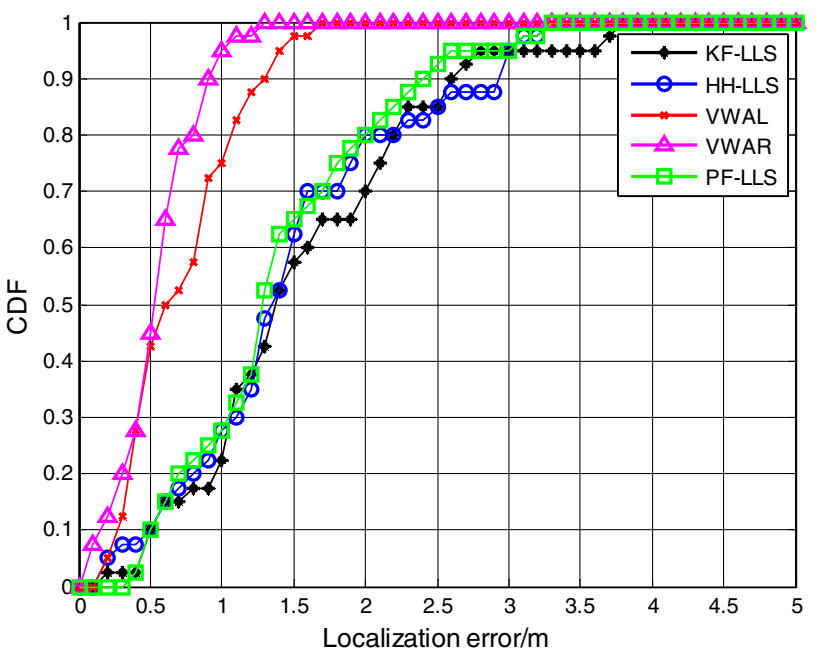

Fig. 15 The cumulative distribution function of localization error in the field experiment

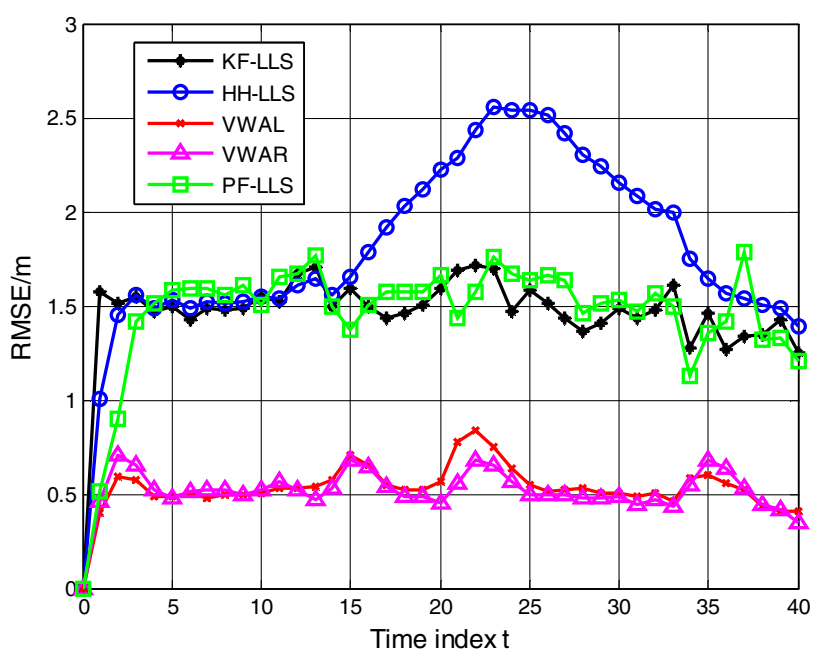

Fig. 16 Comparison of root mean square error

localization accuracy. After the required distance data collection for localization calculation, this algorithm is applied with the collected data using MATLAB to testify the positioning performance in practical environments.

Figure 14 compares the root mean square error of five algorithms. We can observe the RMSE of the KF-LLS, HH-LLS and PF-LLS algorithms are large. By contrast, the localization accuracy of the VWAL and VWAR algorithms are much higher than the previous three algorithms, and the localization accuracy of the VWAR algorithm is slightly higher than the VWAL algorithm.

The cumulative distribution function of localization error in the field experiment is shown in Fig. 15. It shows that the localization error of the PF-LLS, KF-LLS, HH-LLS, VWAL algorithms are $3.3,3.7,3.4$ and $1.7 \mathrm{~m}$ when the cumulative distribution function is close to 1 , while the localization error 


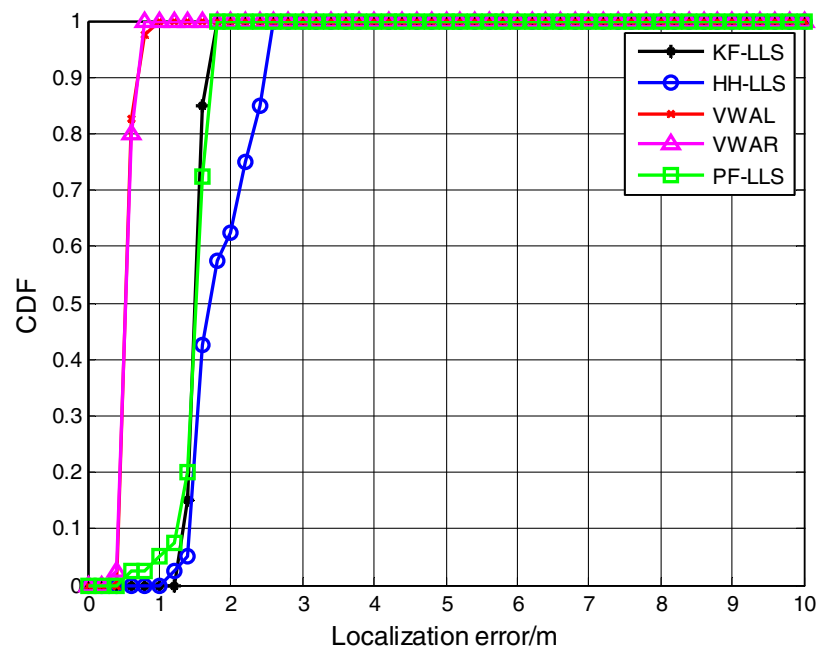

Fig. 17 The cumulative distribution function of localization error

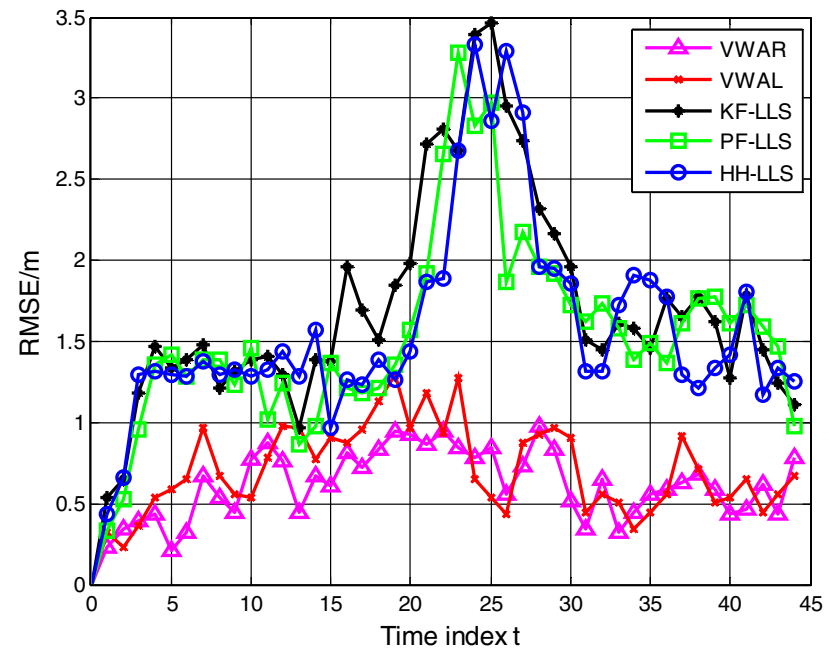

Fig. 18 Comparison of root mean square error in the error in the $6.5 \mathrm{~m} \times 6.5 \mathrm{~m}$ environment

of the VWAR algorithm is $1.25 \mathrm{~m}$. The 90-percentile of localization error of the KF-LLS, HH-LLS, PF-LLS, VWAL and VWAR algorithms are less than 2.6, 2.9, 2.4, 1.3 and 0.85 $\mathrm{m}$. It can be seen the VWAR algorithm has the highest localization accuracy, and the localization accuracy of the VWAR and VWAL algorithms are much higher than the PF-LLS, KF-LLS and HH-LLS algorithms.

To compare with the field experiment, we do the simulation in the same environment. In Figs. 16 and 17, it can be obviously observed that the proposed VWAR and VWAL algorithms own higher localization accuracy in comparing with other methods. The trend of the simulation result is similar to the field experiment. However the localization accuracy of the simulation is higher than the field experiment. This is because the error is small in simulation, and the interference factors are varied in field experiment.

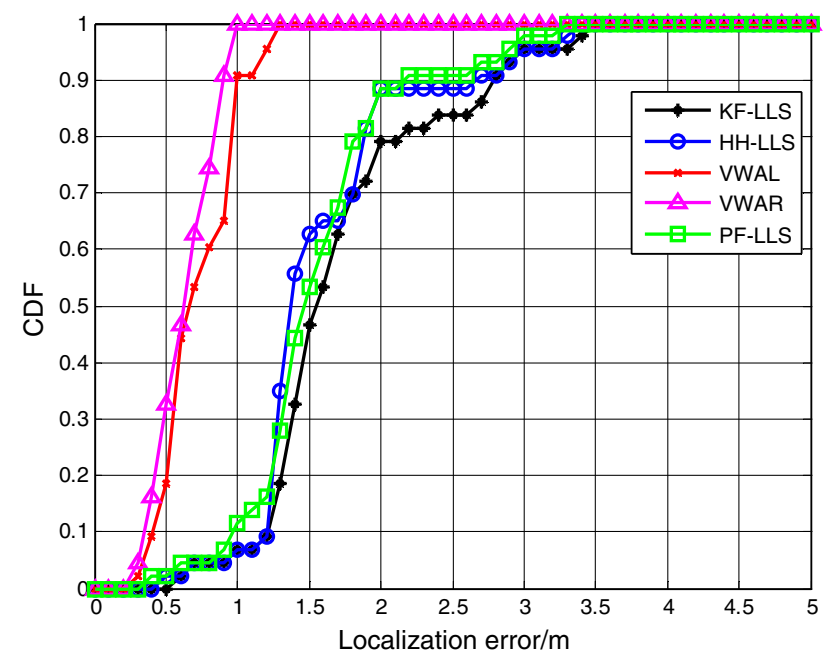

Fig. 19 The cumulative distribution function of localization $6.5 \mathrm{~m} \times$ $6.5 \mathrm{~m}$ environment

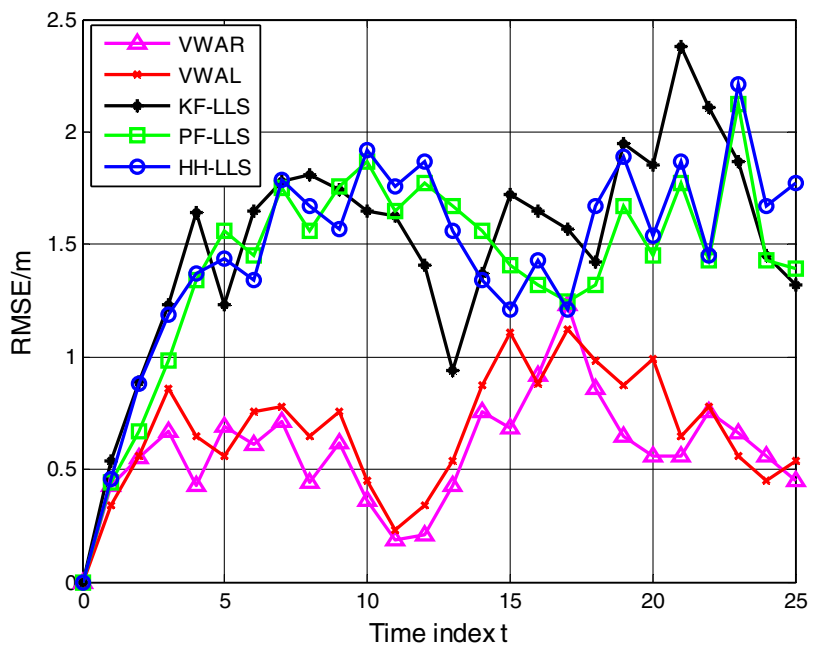

Fig. 20 Comparison of root mean square error in the $2.3 \mathrm{~m} \times 8.5 \mathrm{~m}$ environment

So as to testify the localization performance of the proposed algorithm under other practical applied environments, we deploy the nodes in a $6.5 \mathrm{~m} \times 6.5 \mathrm{~m}$ environment (square room) and a $2.3 \mathrm{~m} \times 8.5 \mathrm{~m}$ environment (long corridor).

As the Figs. 18 and 19 show, the proposed algorithm can get a better performance in compared with other methods in the $6.5 \mathrm{~m} \times 6.5 \mathrm{~m}$ environment. The result in the $6.5 \mathrm{~m} \times 6.5 \mathrm{~m}$ environment is close to the result in $5 \mathrm{~m} \times 7 \mathrm{~m}$ environment. In Fig. 19, we show that the localization errors of the PF-LLS, KF-LLS, HH-LLS, VWAL and VWAR algorithms are 3.25, $3.5,3.39$ and 1.35 and $1.1 \mathrm{~m}$ when the cumulative distribution function is close to 1 .

Figures 20 and 21 show the performance of the methods in $2.3 \mathrm{~m} \times 8.5 \mathrm{~m}$ environment (long corridor). As shown in Fig. 20, the localization error of the proposed methods are less than $1 \mathrm{~m}$ in most situations. Figure 21 shows that VWAR 


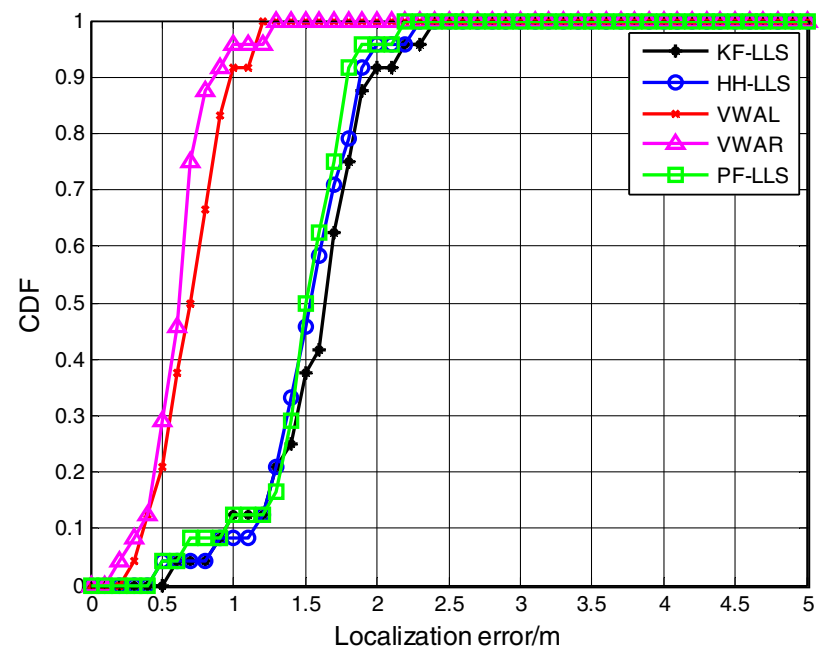

Fig. 21 The cumulative distribution function of localization error in the $2.3 \mathrm{~m} \times 8.5 \mathrm{~m}$ environment

and VWAL have an excellent performance with 90-percentile localization error at 0.8 and $0.9 \mathrm{~m}$ respectively.

\section{Conclusion}

This paper presents a novel localization algorithm for NLOS mobile node based on voting selection mechanisms. Firstly the algorithm makes use of a measurement data preprocessing method based on voting selection mechanisms in order to filter the initial distance measurement values, thus eliminating the adverse impacts on localization calculation caused by the NLOS distance measurements with large NLOS errors. Then the reserved reliable measurement data errors after voting selection are further mitigated through weighting, probabilistic data association and $\mathrm{KF}$ process. Finally, the localization calculation is finished with the filtered distance values using linear least squares method based on reference node selection. The simulation results and the field experiments show that the proposed algorithm has higher localization accuracy and is robust to the NLOS errors in different NLOS environments.

Acknowledgments This work was supported in part by National Natural Science Foundation of China under Grant No. 61273078, 61203216, 61403068 the Fundamental Research Fund for the Central Universities of China No.120404021, N130323004, N130323002, N140403005 Scientific Research Fund of Hebei Provincial Education Department (Z2014078) and the NEUQ internal funding (XNB201509, XNB201510).

Open Access This article is distributed under the terms of the Creative Commons Attribution 4.0 International License (http://creativecomm ons.org/licenses/by/4.0/), which permits unrestricted use, distribution, and reproduction in any medium, provided you give appropriate credit to the original author(s) and the source, provide a link to the Creative Commons license, and indicate if changes were made.

\section{References}

1. Guvenc, I., \& Chong, C. C. (2009). A survey on TOA based wireless localization and NLOS mitigation techniques. IEEE Communications Surveys \& Tutorials, 11(3), 107-124.

2. Shen, Jian, Tan, Haowen, Wang, Jin, Wang, Jinwei, \& Lee, Sungyoung. (2015). A novel routing protocol providing good transmission reliability in underwater sensor networks. Journal of Internet Technology, 16(1), 171-178.

3. Shengdong, Xie, \& Yuxiang, Wang. (2014). Construction of tree network with limited delivery latency in homogeneous wireless sensor networks. Wireless Personal Communications, 78(1), 231246.

4. Guo, Ping, Wang, Jin, Li, Bing, \& Lee, Sungyoung. (2014). A variable threshold-value authentication architecture for wireless mesh networks. Journal of Internet Technology, 15(6), 929-936.

5. Chan, Y. T., Tsui, W. Y., So, H. C., \& Ching, P. C. (2006). Time-of-arrival based localization under NLOS conditions. IEEE Transactions on Vehicular Technology, 55(1), 17-24.

6. Gustafsson, F., \& Gunnarsson, F. (2005). Mobile positioning using wireless networks: Possibilities and fundamental limitations based on available wireless network measurements. IEEE Signal Processing Magazine, 22(4), 41-52.

7. Masazade, E., Fardad, M., \& Varshney, P. K. (2012). Sparsitypromoting extended Kalman filtering for target tracking in wireless sensor networks. IEEE Signal Processing Letters, 19(12), 845-848.

8. Wylie, M. P., \& Holtzman, J. (1996). The non-line-of-sight problems in mobile location estimation. IEEE International Conference on Universal Personal Communications, 2, 827-831.

9. Wann, C. D., \& Lin, M. H. (2004). Data fusion methods for accuracy improvement in wireless location systems. Wireless Communications and Networking Conference, 1, 471-476.

10. Chan, Y. T., \& Ho, K. C. (1994). A simple and efficient estimator for hyperbolic location. IEEE Transactions on Signal Processing, 42(8), 1905-1915.

11. Chen, P. C. (1999). A non-line-of-sight error mitigation algorithm in location estimation. Wireless Communication and Networking Conference, 1, 316-320.

12. Kirubarajan, T., \& Bar-Shalom, Y. (2004). Probabilistic data association techniques for target tracking in clutter. Proceedings of the IEEE, 92(3), 536-557.

13. Wang, J., Li, L., \& Hu, H. (2006). A new approach for cellular wireless location in NLOS situations. IEEE International Conference on Communications and Networking, pp. 1-5.

14. Mazuelas, S., Lago, F. A., \& Blas, J. (2009). Prior NLOS measurement correction for positioning in cellular wireless networks. IEEE Transactions on Vehicular Technology, 58(5), 2585-2591.

15. Venkatesh, S., \& Buehrer, R. M. (2006). A linear programming approach to NLOS error mitigation in sensor networks. 5th International Conference on Information Processing in Sensor Networks, pp. 301-308.

16. Venkatraman, S., Caffery, J., \& You, H. R. (2004). A novel TOA location algorithm using LOS range estimation for NLOS environments. IEEE Transactions on Vehicular Technology, 53(5), $1515-1524$.

17. Wang, X., Wang, Z., \& O'Dea, B. (2003). A TOA-based location algorithm reducing the errors due to non-line-of-sight (NLOS) propagation. IEEE Transactions on Vehicular Technology, 52(1), 112-116.

18. Chen, H., Wang, G., \& Wang, Z. (2012). Non-line-of-sight node localization based on semi-definite programming in wireless sensor networks. IEEE Transactions on Wireless Communications, 11(1), $108-116$

19. Liao, J. F., \& Chen, B. S. (2006). Robust mobile location estimator with NLOS mitigation using interacting multiple model algorithm. 
IEEE Transactions on Wireless Communications, 5(11), 30023006.

20. Chen, C. L., \& Feng, K. T. (2005). An efficient geometryconstrained location estimation algorithm for NLOS environments. 2005 IEEE International Conference on Wireless Networks, Communications and Mobile Computing, vol. 1, pp. 244-249.

21. Abumansoor, O., \& Boukerche, A. (2012). A secure cooperative approach for non-line-of-sight location verification in VANET. IEEE Transactions on Vehicular Technology, 61(1), 275-285.

22. Yu, K., \& Dutkiewicz, E. (2013). NLOS identification and mitigation for mobile tracking. IEEE Transactions on Aerospace and Electronic Systems, 49(3), 1438-1452.

23. Hammes, U., \& Zoubir, A. M. (2011). Robust MT tracking based on M-estimation and interacting multiple model algorithm. IEEE Transactions on Signal Processing, 59(7), 3398-3409.

24. Lin, X., Ju, L., \& Chen, S. (2008). NLOS error identification and range approximation technique in cellular networks. 4th International Conference on Wireless Communications, Networking and Mobile Computing, pp. 1-4.

25. Cheng, L., Wu, H., Wu, C. D., \& Zhang, Y. Z. (2013). Indoor mobile localization in wireless sensor network under unknown NLOS errors. International Journal of Distributed Sensor Networks, pp. 1-9.

26. Wang, Z. H., \& (Reza) Zekavat, Seyed, A. (2012). Omnidirectional mobile NLOS identification and localization via multiple cooperative nodes. IEEE Transactions on Mobile Computing, 11(12), 2047-2059.

27. Wu, S. X., Li, J. P., \& Liu, S. Y. (2013). Improved localization algorithms based on reference selection of linear least squares in LOS and NLOS environments. Wireless Personal Communications, 68 , 187-200.

28. Cheng, L., Wang, Y., Wu, H., Hu, N., \& Wu, C. D. (2015). Nonparametric location estimation in rough wireless environments for wireless sensor network. Sensors and Actuators A, 224, 57-64.

29. Wann, C. D., \& Hsueh, C. S. (2007). NLOS mitigation with biased Kalman filters for range estimation in UWB systems. IEEE TENCON, 2007, 1-4.

30. Ni, L., Gao, S., \& Xue, L. (2006). Visual tracking in cluttered environments: Using the visual probabilistic data association filter [J]. IEEE Transactions on Robotics, 22(6), 1292-1297.

31. Lee, S. W., Ha, R., \& Cha, H. J. (2014). IEEE 802.15.4a CSS-based mobile object locating system using sequential mote carlo method. Computer Communication, 38, 13-25.

32. Kang, H. G., Seo, G. W., \& Lee, J. H. (2009). Error compensation for CSS-based localization system. Proceedings of the World Congress on Engineering and Computer Science 2009, vol. II.

33. Hur, H., \& Ahn, H. S. (2013). Discrete-time $\mathrm{H} \infty$ filtering for mobile robot localization using wireless sensor network. IEEE Sensors Journal, 13(1), 245-252.

34. Wang, J., Gao, Q. H., Yu, Y., Wang, H. Y., \& Jin, M. L. (2012). Toward robust indoor localization based on bayesian filter using chirp-spread-spectrum ranging. IEEE Transactions on Industrial Electronics, 59(3), 1622-1629.

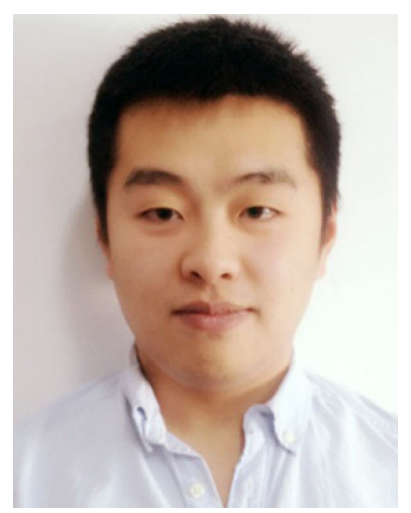

Nan Hu received B.S. degree in electric engineering and automation from Shenyang Jianzhu University, Shenyang, China, in 2010, the M.S. degree in system engineer from Northeastern University, Shenyang, China, in 2012. He is pursuing the Ph.D. degree in pattern recognition and intelligent system from Northeastern University, Shenyang, China. His research interests include wireless sensor networks and distributed system.

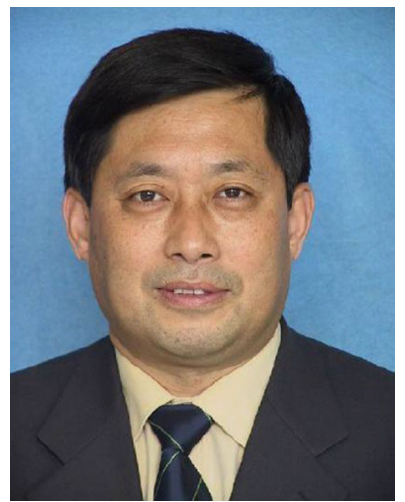

Chengdong Wu received M.S. degree in automatic control from Tsinghua University, Beijing, China, in 1988. He received Ph.D. degree in automatic control from North-eastern University, Shenyang, China, in 1994. From 1995 to 1997 , he worked as a postdoctoral researcher in University of Wales, U.K. From 2001 to 2002, he worked as senior visiting professor in University of Bedfordshire, U.K. Now, he is a full professor in Northeastern University. His current research interests include wireless sensor networks and robot.

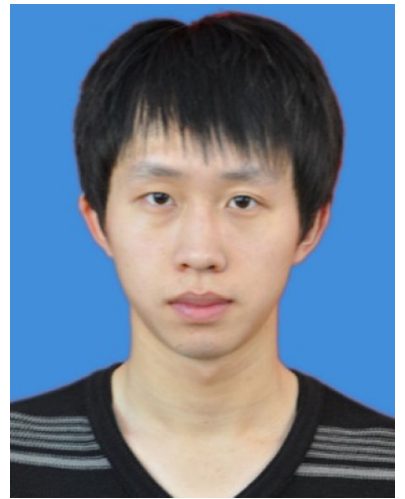

Pengda Liu received B.S. degree in automation from Northeastern University, Shenyang, China, in 2012, the M.S. degree in the pattern recognition and intelligent system from Northeastern University, Shenyang, China, in 2010. He is pursuing the Ph.D. degree in automatic control from Northeastern University, Shenyang, China. His research interests include distributed and parallel computing. 


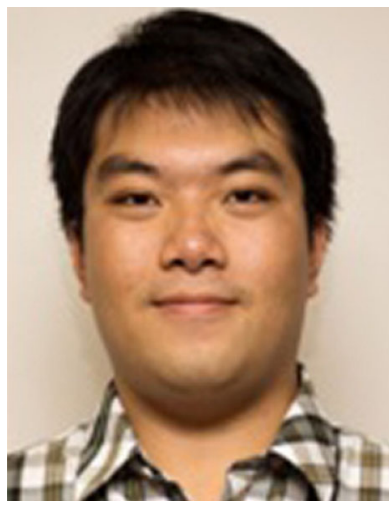

Hao Wu received B.S. degree in computer science and technology from Northeastern University, Shenyang, China, in 2012, the M.S. degree in the engineering faculty, University of Sydney, NSW, Australia, in 2013. He is pursuing the Ph.D. degree in the engineering faculty, University of Sydney, NSW, Australia. His research interests include distributed and parallel computing.

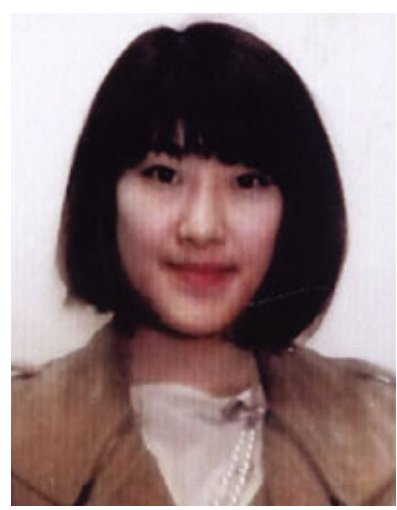

Boya Wu received B.S. degree in International Relations and International Communication from the Australian National University, Canberra, Australia in 2010, the M.S. degree in Asian Pacific from the Australian National University, Canberra, Australia in 2011. Her research areas include western linguistic study, English speaking countries culture and civilization and Asian Pacific Study.

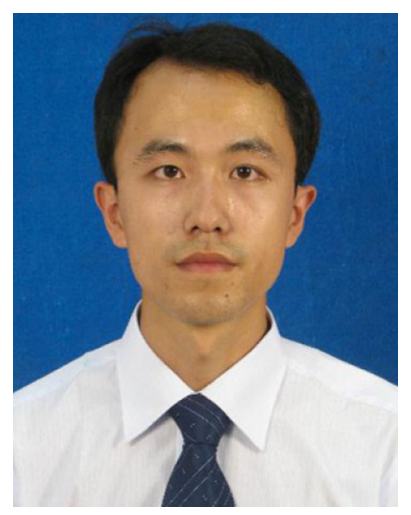

Long Cheng received B.S. degree in automatic control from Qingdao University of Science and Technology, Qing-dao, China, in 2008, the M.S. degree in pattern recognition and intelligent system from Northeastern University, Shenyang, China, in 2010. He received Ph.D. degree in pattern recognition and intelligent system from North-eastern University, Shenyang, China, in 2013. Now, he is an assistant professor in Northeastern University. His research interests include wireless sensor networks and distributed system. 